\title{
Anisotropic Landau-Lifshitz-Gilbert models of dissipation in qubits
}

\author{
Philip J. D. Crowley and A. G. Green \\ London Centre for Nanotechnology, University College London, Gordon St, London, WC1H OAH, United Kingdom
}

(Received 2 March 2015; published 9 December 2016)

\begin{abstract}
We derive a microscopic model for dissipative dynamics in a system of mutually interacting qubits coupled to a thermal bath that generalizes the dissipative model of Landau-Lifshitz-Gilbert to the case of anisotropic bath couplings. We show that the dissipation acts to bias the quantum trajectories towards a reduced phase space. This model applies to a system of superconducting flux qubits whose coupling to the environment is necessarily anisotropic. We study the model in the context of the D-Wave computing device and show that the form of environmental coupling in this case produces dynamics that are closely related to several models proposed on phenomenological grounds.
\end{abstract}

DOI: 10.1103/PhysRevA.94.062106

\section{INTRODUCTION}

The Landau-Lifshitz-Gilbert equation [1,2] provides a phenomenologically motivated description of the stochastic, dissipative evolution of a spin system. Conceived as a model for an open magnetic system, the dynamics consists of two terms corresponding to precessing, Hamiltonian evolution, and noisy, relaxing, dissipative evolution. This form of dissipation corresponds to the classical limit of a spin system coupled isotropically to a bosonic bath.

The behavior of a superconducting quantum device can be mapped to spin dynamics [3]. In the case of a flux qubit, the low-energy dynamics map on to those of a spin-1/2 magnetic moment, and an isolated qubit will exhibit only the Larmor precession described by a dissipation-free Landau-LifshitzGilbert equation; the qubit wave function evolves such that the absolute amplitudes of the two energy eigenstates remain constant while the phase between them changes at a constant rate.

This relationship suggests that qubits coupled to the environment may display the same dissipative behavior as magnetic moments, governed by the full dissipative LandauLifshitz-Gilbert equation. This model has been used to model an extended array of superconducting qubits in Ref. [4] while related vector models have been used in Refs. [5-7].

However, the Landau-Lifshitz-Gilbert equation describes the dissipative dynamics of a two-level system that is exposed to isotropic environmental coupling, i.e., identical baths coupled to the $\hat{s}_{x}, \hat{s}_{y}$, and $\hat{s}_{z}$ operators. For qubits, these operators may have different physical origins and hence will couple differently to noise. Thus, the dissipation will also be anisotropic, as the stochastic noise and dissipative terms are related by the fluctuation dissipation relation. As a result, systems, such as flux qubits, with anisotropic couplings have corresponding anisotropies in the dissipation and noise.

Due to the physical geometry of the superconducting flux qubit, stray flux, and other environmental effects couple to the $\hat{s}_{z}$ operator. The anisotropy of this environmental coupling introduces qualitatively new features into the system's dynamics. We show the existence of a regime where the qubit dynamics are typically confined to a low-dimensional submanifold of the full Hilbert space.

The dissipative dynamics of a two-level system has been studied extensively [3]. In this work, we use a Keldysh path integral [8-10] to derive a Langevin [11,12] description of the dynamics of a flux qubit accounting for the anisotropic coupling to the environment. This extends the previous work on dissipative spin models [13-22].

An accurate model for the dissipative dynamics of a flux qubit can be used to assess the capabilities of putative quantum technologies. We apply our model to the D-Wave computing machine, which consists of a large array of controllable flux qubits. Extensive analysis has sought to correlate the behavior of this machine with various quantum and classical models [4-7,23-26]. Since classical dynamics correspond to a particular restriction upon fully quantum dynamics, the effectiveness of this approach is dependent upon identifying the appropriate restrictions that correspond to the classical limit. We show in an appropriate strong coupling limit the biasing of trajectories in the anisotropic Langevin equation allows one to obtain dynamics reminiscent of the heuristic models of Refs. [5,7,24].

\section{ANISOTROPIC DISSIPATION OF FLUX QUBITS}

A superconducting flux qubit will couple to its environment in various ways. The environmental degrees of freedom may consist of charge fluctuations, flux noise, coupling to nearby spins, or trapped vortices. When it is not possible to interrogate such environmental subsystems, the information contained in the state of these subsystems is lost from the system of interest - the qubit. This decoherence inhibits the ability of a qubit to remain in a given state indefinitely with good fidelity, and in turn limits the ability of an array of qubits to sustain entanglement.

This loss of unitary evolution generates dissipative dynamics that, over sufficiently long times, drive the system towards certain equilibrium states, or dynamical fixed points. In this sense, the effects of decoherence are inherently inhomogeneous over the system's Hilbert space, they affect different states in different ways, driving them towards different fixed points or along different paths to such fixed points.

We anticipate that a system exchanging energy with an environmental bath will relax to a fixed point given by the Gibbs state. There remains, however, a plurality of dynamics that result in the system relaxing to this state, and we expect that different baths and couplings with different physical origins will result in different relaxation dynamics. This is relevant to controlled quantum systems in which the dynamics of interest occur before the system has relaxed to thermal equilibrium, 
but not necessarily on timescales where dissipation can be neglected.

The Landau-Lifshitz-Gilbert describes the dissipative dynamics of a two-level system with isotropic coupling to the environment. It is often quoted in one of two equivalent forms:

$$
\begin{array}{r}
\dot{\mathbf{s}}+\mathbf{s} \times[(\mathbf{B}+\boldsymbol{\eta})-\gamma \dot{\mathbf{s}}]=0, \\
\dot{\mathbf{s}}+\frac{1}{1+s^{2} \gamma^{2}} \mathbf{s} \times[(\mathbf{B}+\boldsymbol{\eta})+\gamma \mathbf{s} \times(\mathbf{B}+\boldsymbol{\eta})]=0 .
\end{array}
$$

These are nonlinear differential equations in a vector $\mathbf{s}$ of magnitude $s$ that parametrizes a qubit spin coherent state $\hat{\mathbf{s}}|\mathbf{s}\rangle=\mathbf{s}|\mathbf{s}\rangle$. The equation is stated here in terms of an effective magnetic field, B. More generally, the magnetic field may be replaced by an appropriate derivative of the spin Hamiltonian, $\mathbf{B}=-\nabla_{\mathbf{s}} H(\mathbf{s}) . \eta$ describes a stochastic noise that satisfies the fluctuation dissipation relation; $\left\langle\eta_{\alpha}(\omega) \eta_{\beta}\left(\omega^{\prime}\right)\right\rangle=$ $\gamma \omega \operatorname{coth}\left(\frac{\omega}{2 T}\right) \delta_{\alpha \beta} \delta\left(\omega+\omega^{\prime}\right)[27]$.

This description closely resembles the phenomenological Bloch equations used to model nuclear magnetization, the difference being the Landau-Lifshitz-Gilbert equation describes a single spin and not an ensemble average, thus the decay must preserve the spin $|\mathbf{s}|=s$. Neglecting zero-point fluctuations, this Langevin equation describes the dissipative evolution of a single qubit in the presence of a magnetic field, $\mathbf{B}$, and coupled isotropically to its environment.

The dynamics of a system of multiple interacting qubits may be described by a set of coupled Landau-Lifshitz-Gilbert equations. These dynamics are general and constitute a restriction to product states. Coherent dynamics are permitted for individual spins in these states, but there is no entanglement between spins. In this sense the equations correspond to the classical limit of the system.

Although originally developed to describe spins that are isotropically susceptible to noise, the Landau-Lifshitz-Gilbert equations have been used to model the classical dynamics of dissipative qubits [4], while other authors have also made use of similar vector models [7,24]. However, as argued above, the physics underlying superconducting flux qubits implies that noise and dissipation will be anisotropic. While the microscopic origins of flux qubit decoherence are not fully understood [28], many environmental interactions are often modeled using linear $\hat{s}_{z}$ couplings [3]. These include flux noise and coupling to impurity spin [28-31] or boson [32,33] degrees of freedom, and have been shown to be significant contributions to decoherence [34]. While in the context of the D-Wave computing machine it has been argued that linear $\hat{s}_{z}$ coupling is the correct minimal model [35]. Without loss of generality any linear coupling can be chosen to be $\hat{s}_{z}$ and whether longitudinal or transverse. Due to the close relationship between the damping and noise terms enforced by the fluctuation dissipation relation, this cannot be remedied solely by an appropriate adjustment to the noise term; an adjustment to the noise term effects the damping to term in a manner that is difficult to guess.

\section{DYNAMICAL EQUATION WITH ANISOTROPIC DISSIPATION}

Here we follow the approach of Ref. [8] to find the partition function $Z=\prod_{i} \int D\left[\mathbf{s}_{i}\right] \mathrm{e}^{i S\left[\left\{\mathbf{s}_{i}\right]\right\}}$ for an open quantum system, and evaluate the dynamics of a qubit using a stationary phase approximation. In a closed system, the action of the spins is given by

$$
S_{0}=\int_{\mathcal{C}} d t L_{0}=\int_{\mathcal{C}} d t\left(\sum_{i} \dot{\mathbf{s}_{i}} \cdot \mathbf{A}_{i}-H\left(\left\{\mathbf{s}_{i}\right\}\right)\right)
$$

where $\mathbf{A}_{i}=\frac{1-\cos \theta_{i}}{\sin \theta_{i}} \hat{\boldsymbol{\phi}}$ is the single monopole vector potential. This action describes the precession of a spin around the axis of the magnetic field. When the spin is coupled to a bath of oscillators through the $s_{z}$ component, the action becomes

$$
S=S_{0}+\sum_{i, \alpha} \int_{\mathcal{C}} d t\left[s_{i, z} g_{i, \alpha} x_{\alpha}+\frac{m}{2}\left(\dot{x}_{\alpha}^{2}-\omega_{\alpha}^{2} x_{\alpha}^{2}\right)\right]
$$

We use Keldysh field theory to find the dynamics of this system. This provides a methodology for treating an open quantum system, enforcing the necessary fluctuation dissipation relation between the stochastic noise and deterministic damping terms induced by decoherence.

A state vector is sufficient to define the behavior of a closed system. Nonequilibrium, open quantum systems require an ensemble of state vectors, or a density matrix, because of the loss of information to the bath. Evolving a density matrix $\hat{\rho}$ requires both premultiplication and postmultiplication by the time evolution operator $\hat{U}\left(t_{1}, t_{2}\right)=\mathcal{T} \exp \left[i \int_{t_{2}}^{t_{1}} d t \hat{H}(t)\right]$. Thus $\hat{\rho}_{t}=\hat{U}(t, 0) \hat{\rho}_{0} \hat{U}(0, t)$ in contrast to the time evolution of a state vector, which requires only one time evolution operator $\left|\psi_{t}\right\rangle=\hat{U}(t, 0)\left|\psi_{0}\right\rangle$. This gives rise to a doubling of the degrees of freedom in the Keldysh theory of open systems compared to those required to describe the evolution of a closed system. These degrees of freedom correspond to the forward and backward branches of the Keldysh contour, $\mathcal{C}$, over which Eqs. (2) and (3) are integrated.

It is useful in calculations to separate diagonal and offdiagonal contributions to the density matrix. The former are described by the sum of fields on the forwards and backwards Keldysh contour, and the latter by their difference. These fields are usually called the classical and quantum fields respectively. Expanding in the quantum fields generates a series of higher-order quantum corrections to the classical dynamics. Performing this rotation, and expanding to first order in the quantum fields results in each spin having a total action $S=S_{0}+S_{\text {diss }}$

$$
\begin{aligned}
S= & \int_{\mathbb{R}} d t\left[\sum_{i} 2 \mathbf{s}_{i}^{q} \cdot\left(\frac{\partial L_{0}}{\partial \mathbf{s}_{i}}-\frac{d}{d t} \frac{\partial L_{0}}{\partial \dot{s}_{i}}\right)\right. \\
& +2 \sum_{i, \alpha} g_{i, \alpha}\left(s_{i, z} x_{\alpha}^{q}+s_{i, z}^{q} x_{\alpha}\right)+\sum_{\alpha}\left(x_{\alpha} x_{\alpha}^{q}\right) \\
& \left.\times\left(\begin{array}{cc}
0 & {\left[D_{\alpha}^{A}\right]^{-1}} \\
{\left[D_{\alpha}^{R}\right]^{-1}} & {\left[D_{\alpha}^{-1}\right]^{K}}
\end{array}\right)\left(\begin{array}{c}
x_{\alpha} \\
x_{\alpha}^{q}
\end{array}\right)\right] .
\end{aligned}
$$

The first term in Eq. (4) encodes the Hamiltonian (closed system) dynamics of each spin. The remaining terms encode the dissipative dynamics induced by interactions with the bath. 


\section{Langevin equation}

The dynamical equation can be obtained from a limit of the Keldysh theory as follows: In an open system the state of bath cannot be interrogated, thus it is integrated out to obtain the dissipative contribution to the action in terms of the spin alone;

$$
S_{\mathrm{diss}}=-\sum_{i} \int_{\mathbb{R}} d t\left[\left(s_{i, z} s_{i, z}^{q}\right)\left(\begin{array}{cc}
0 & D^{A} \\
D^{R} & D^{K}
\end{array}\right)\left(\begin{array}{c}
s_{i, z} \\
s_{i, z}^{q}
\end{array}\right)\right],
$$

where the correlators $D^{A}=\sum_{\alpha} g_{i, \alpha}^{2} D_{\alpha}^{A}$, and similarly for $D^{R}$ and $D^{K}$, are equal for each spin, and defined by the bath spectral function $J(\omega)=\sum_{\alpha} \pi g_{i, \alpha}^{2} / \omega \delta\left(\omega-\omega_{\alpha}\right)$. The equation is made linear in the spin fluctuation variables $s_{z}^{q}$ by a Hubbard-Stratonovich transformation

$$
S_{\mathrm{diss}}=\sum_{i} \int_{\mathbb{R}} d t\left(2 s_{i, z}^{q} D^{R} s_{i, z}+2 s_{i, z}^{q} \eta_{i}-\eta_{i}\left[D^{K}\right]^{-1} \eta_{i}\right),
$$

which introduces the variable $\eta$ that later appears as the stochastic term in the dynamical equations. $\mathbf{s}^{q}$ now act as Lagrange multipliers and can be integrated out of the partition function to obtain

$$
\begin{aligned}
Z= & \prod_{i} \int D\left[\eta_{i}\right] \mathrm{e}^{-i \int \eta_{i}\left[D^{K}\right]^{-1} \eta_{i} d t} \int D\left[\mathbf{s}_{i}\right] \\
& \times \delta\left(\frac{1}{s^{2}} \mathbf{s}_{i} \times \dot{\mathbf{s}}_{i}+\nabla_{\mathbf{s}_{i}} H+\int_{\mathbb{R}} d t^{\prime} D^{R}\left(t-t^{\prime}\right) s_{i, z}\left(t^{\prime}\right) \mathbf{z}+\eta_{i} \mathbf{z}\right)
\end{aligned}
$$

the dynamical equations describing the semiclassical dynamics of the system are then found are then found by taking the saddle points of the path integral. The resulting dynamical equation is

$$
\dot{\mathbf{s}}_{i}=\mathbf{s}_{i} \times\left[\nabla_{\mathbf{s}_{i}} H+\int_{-\infty}^{t} d t^{\prime} \Gamma\left(t-t^{\prime}\right) \dot{s}_{i, z}\left(t^{\prime}\right) \hat{\mathbf{z}}+\eta \hat{\mathbf{z}}\right],
$$

where the Keldysh correlator $D^{K}\left(t-t^{\prime}\right)=-2 i\left\langle\eta_{i}(t) \eta_{i}\left(t^{\prime}\right)\right\rangle$ defines the Gaussian noise correlations. Evaluating this via the fluctuation dissipation relation $D^{K}=\left(D^{R}-D^{A}\right) \operatorname{coth}\left(\frac{\omega}{2 T}\right)$ one arrives at

$$
\left\langle\eta_{i}(t) \eta_{j}\left(t^{\prime}\right)\right\rangle=\frac{\delta_{i j}}{4 \pi} \int_{0}^{\infty} d \omega J(\omega) \operatorname{coth}\left(\frac{\omega}{2 T}\right) \cos \left[\omega\left(t-t^{\prime}\right)\right],
$$

while the retarded correlator defines the damping kernel

$$
\Gamma(t)=\int_{-\infty}^{t} d t^{\prime} D^{R}\left(t^{\prime}\right)=\frac{1}{2 \pi} \int_{0}^{\infty} \frac{d \omega}{\omega} J(\omega) \cos (\omega t) .
$$

In Eq. (8) the bare capacitance has been renormalized by a term generated by the bath in the usual way [3].

Many of the dynamical features of this model can be seen within the Markovian approximation in which the bath memory is neglected. In this regime the dynamics are described by the Langevin equation, which can be expressed in two equivalent forms, where $\boldsymbol{\eta}_{i}=\eta_{i} \hat{\mathbf{z}}$ and $\mathbf{B}_{i}=-\nabla_{\mathbf{s}_{i}} H=\mathbf{h}_{i}+$ $\sum_{j} \mathbf{J}_{i j} \mathbf{s}_{j}+\cdots$, we obtain

$$
\begin{array}{r}
\dot{\mathbf{s}}_{i}+\mathbf{s}_{i} \times\left[\left(\mathbf{B}_{i}+\boldsymbol{\eta}_{i}\right)-\gamma \hat{\mathbf{z}}\left(\hat{\mathbf{z}} \cdot \dot{\mathbf{s}}_{i}\right)\right]=0, \\
\dot{\mathbf{s}}_{i}+\mathbf{s}_{i} \times\left(\mathbf{B}_{i}+\boldsymbol{\eta}_{i}\right)+\gamma \mathbf{s}_{i} \times \hat{\mathbf{z}}\left[\hat{\mathbf{z}} \cdot\left(\mathbf{s}_{i} \times \mathbf{B}_{i}\right)\right]=0 .
\end{array}
$$

The Langevin equation, Eq. (11a), is a stochastic differential equation whose solution is given by an ensemble of pure state trajectories. While it is possible to obtain the many body dynamics within any manifold of quantum states, to obtain the classical dynamics of a many qubit system, we have restricted the pure state trajectories to product states, thus encoding only classical correlations.

The Langevin equations, Eqs. (11a) and (11b), obtained by this method include, by construction, the dissipative and stochastic effects induced by an anisotropic bath. These dynamics are different from those obtained for an isotropic bath [36], Eqs. (1a) and (1b), with important consequences.

\section{DYNAMICS OF THE MODEL}

We now explore the novel dynamics in the presence of anisotropic coupling to the bath in its different parameter regimes. This model, Eqs. (11a) and (11b), describes the dissipative dynamics of a system of interacting, nonentangled flux qubits, with environmental coupling solely through the $\hat{s}_{z}$ operator. There are important differences between the effects of isotropic environmental couplings, Eqs. (1a) and (1b), and anisotropic couplings, Eqs. (11a) and (11b). The energy-conserving dynamics of these two models are the same, consisting of precession about the external field, and they both relax to the same thermal equilibrium distribution. We shall concentrate upon the case where thermal fluctuations are small in the sense that the equilibrium thermal distribution subtends a small solid angle on the Bloch sphere. This requires that $\left\langle\theta^{2}\right\rangle \approx T / B \ll \pi^{2}$, or alternatively $B \gg T$. In this limit, we may sensibly discuss a large deviation from thermal equilibrium and consider the dissipative relaxation to it. These dynamics are very different in the presence of an isotropic, Eqs. (1a) and (1b), and anisotropic coupling, Eqs. (11a) and (11b), to the bath.

The Landau-Lifshitz-Gilbert equation constitute a special case where the Hamiltonian and dissipative dynamics separate in the equations of motion. This separation is clear when written in polar coordinates. For brevity we neglect stochastic terms and let $\mathbf{B} \| \mathbf{z}$, we find

$$
\dot{\theta}=-\frac{\gamma s B}{1+\gamma^{2} s^{2}} \sin \theta, \quad \dot{\phi}=\frac{B}{1+\gamma^{2} s^{2}} .
$$

These equations generate motion in perpendicular directions and there is no interplay between their dynamics.

In contrast, in more general dissipative models there is no such separation of the effects of precession and dissipation, and their interplay remains important. In the Langevin equation for $\hat{s}_{z}$ coupling, Eq. (11b), the system relaxes indirectly, through the interplay of dynamics and the state-dependent modulation of the rate of dissipation. The effect of this interplay is highlighted by the appearance of regions of novel behavior, visible in Fig. 1, such as dissipation-free precession, retrograde motion, and effective dimensional reduction. 

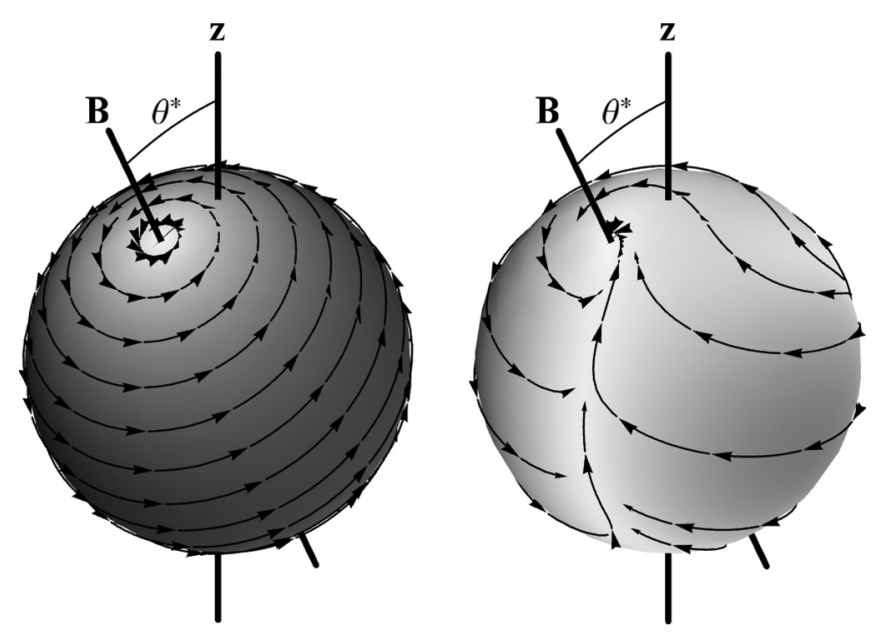

(a) $\theta^{*}=\frac{\pi}{8}, \gamma s=0.6$

(b) $\theta^{*}=\frac{\pi}{8}, \gamma s=6$

FIG. 1. Dynamics of spins in the presence of anisotropic dissipation. The direction and rate of evolution over the sphere surface are indicated by the streamlines and grayscale density, darker regions indicate faster evolution. (a) For weak anisotropic coupling the state precesses similarly to isotropic coupling. (b) For strong anisotropic coupling dynamics is markedly different. The system rapidly relaxes to a reduced $O(2)$ manifold where it undergoes constrained dynamics.

To discuss the dynamics of the $\hat{s}_{z}$-coupling model, it is useful to introduce the timescales $\tau_{\mathrm{p}}^{-1}=B=|\mathbf{B}|$ and $\tau_{d}^{-1}=$ $\gamma s B \sin ^{2} \theta^{*}$ where $\theta^{*}$ is the polar angle of the field $\mathbf{B}$ from z. $\tau_{\mathrm{p}}$ and $\tau_{d}$, which are characteristic of the precessional motion and dissipative motion, respectively. In the limits where these scales are widely separated, the system's behavior is dominated by the faster dynamics on short timescales, while some effective dynamics emerge on longer timescales.

\section{A. Weak coupling limit, $\tau_{\mathrm{p}} \ll \tau_{d}$}

For weak coupling, the system's behavior remains dominated by the precession found in the fully closed system dynamics. As shown in Fig. 1(a), the spin will generally perform many rotations about the magnetic field, $\mathbf{B}$, before reaching the neighborhood of the ground state. This allows the dissipation and noise to be averaged over these rotations. In this regime the Langevin equation may be approximated by a Landau-Lifshitz-Gilbert equation

$$
\dot{\mathbf{s}}+\mathbf{s} \times\left[\left(\mathbf{B}+\boldsymbol{\eta}_{\mathrm{eff}}\right)+\gamma_{\mathrm{eff}} \mathbf{s} \times\left(\mathbf{B}+\boldsymbol{\eta}_{\mathrm{eff}}\right)\right]=0,
$$

where $\gamma_{\mathrm{eff}}=\frac{1}{2} \gamma \sin \theta^{*}$ is the effective dissipation, This results in dynamics characteristically similar to the isotropic case [Eqs. (1a) and (1b)], thus the limit $\tau_{\mathrm{p}} \ll \tau_{d}$ offers no novel dynamics.

\section{B. Strong coupling limit, $\tau_{\mathrm{p}} \gg \tau_{d}$}

In the limit $\tau_{\mathrm{p}} \gg \tau_{d}$, when the effects of anisotropy show up most profoundly, the system's dynamics are dominated by the dissipative term. This term is state dependent and drives rotation about the $\mathbf{z}$ axis, however, the dissipation goes to zero when $\mathbf{B}, \mathbf{s}$, and $\mathbf{z}$ are coplanar.

Exactly how this reveals itself in the dynamics depends upon the bath memory and temperature, though the net effect is similar in all cases: we see a separation of timescales and a reduction of the full $O(3)$ qubit dynamics to effective $O(2)$ dynamics characterized by fast decay towards, or oscillation around, a reduced manifold.

Markovian approximation. In the first instance, it is easiest to analyze our model within an Markovian approximation. Separating Eq. (11b) into the slow $\theta$ and fast $\phi$ dynamics we obtain

$$
\begin{aligned}
& \dot{\theta}=-B \sin \theta^{*} \sin \phi \\
& \dot{\phi}=B\left(\cos \theta^{*}-\sin \theta^{*}[\cos \phi \cot \theta+s \gamma \sin \theta \sin \phi)\right]+\eta
\end{aligned}
$$

where $\phi$ typically relaxes with a characteristic timescale

$$
\tau_{\phi}=\frac{1}{\gamma s B \sin \theta \sin \theta^{*}}
$$

during which time $\theta$ makes a very small change. Thus we can treat the appearance of $\phi$ in the $\theta$ dynamics as a stochastic variable sampling the quasistatic distribution of the $\phi$ dynamics. This quasi thermal distribution is given approximately by $p(\phi) \propto \exp \left[-A(\theta) \cos \left(\phi-\phi^{*}\right)\right]$ where $A(\theta)^{-1}=$ $\gamma T \tau_{\phi} \cos \phi^{*}$ and $\tan \phi^{*}=\sin \left(\theta-\theta^{*}\right) /\left(s \gamma \sin ^{2} \theta \sin \theta^{*}\right)$. General effective dynamics in the slow $\theta$ variable are obtained in Appendix A, the high- and low- $T$ cases are discussed here.

For $T \ll B$ the system quickly relaxes to a state in which the $\phi$ distribution is sharply peaked around the dynamical fixed point close to $\phi^{*} \approx 0$. This confining behavior, as shown in Fig. 1(b) occurs when the dissipative dynamics drive the system towards a one-dimensional manifold on the fast timescale $\tau_{d}$, after which a much slower interplay between the Hamiltonian and dissipative dynamics sees the system relax to its ground state. These latter dynamics are described by

$$
\dot{\theta}=\frac{B \sin \left(\theta^{*}-\theta\right)}{s \gamma \sin ^{2} \theta}+\eta^{\prime},
$$

where the noise $\langle\eta(t)\rangle=0$ has correlations $\left\langle\eta^{\prime}(t) \eta^{\prime}\left(t^{\prime}\right)\right\rangle=$ $2 \gamma T\left(\tau_{\phi} B \sin \theta^{*}\right)^{2} \delta\left(t-t^{\prime}\right)$. In this limit the stochastic effect of the bath is weak (in the sense that thermal fluctuations subtend only a small angle on the Bloch sphere), whereas it strongly biases the trajectories to dissipate energy. In this manner the effect is akin to the trajectory ensemble approach [37] with a transition to dynamics confined to $\phi \approx \phi^{*}$. Due to the known relationship between these dynamics [38-40] this Langevin equation can also be related to a Monte Carlo $O(2)$ model on appropriate timescales.

For $T \gg B$ the noise is sufficiently strong that $\phi$ makes large excursions away from the dynamical fixed point. However, due to the separation of timescales, in both cases the long-time dynamics are captured by an effective theory in the slow variable $\theta$. This constitutes a dissipative reduction of the phase space from $O(3)$ to effective $O(2)$ dynamics. In the $T \gg B$ limit, this reduced dynamics is described by

$$
\dot{\theta}=\frac{B^{2} \sin \left(\theta^{*}-\theta\right) \sin \theta^{*}}{2 T \gamma \sin \theta}+\eta^{\prime},
$$

where the noise has correlations $\left\langle\eta^{\prime}(t) \eta^{\prime}\left(t^{\prime}\right)\right\rangle=$ $\tau_{\phi}\left(B \sin \theta^{*}\right)^{2} \delta\left(t-t^{\prime}\right)$.

The limit in which the dynamics become Markovian is subtle. Often Markovian dynamics can be obtained by 
assuming an Ohmic bath $J(\omega)=4 \gamma \omega$. When the temperature is much greater than the characteristic frequency of the system (the frequency at which the bath dynamics are sampled) the bath falls in its classical limit, $\operatorname{coth}(\omega / 2 T) \rightarrow 2 T / \omega$, so that Eq. (9) yields a $\delta$-correlated noise. In this regime, however, thermal motion generally dominates, in the sense that thermal fluctuations cover the entire Bloch sphere; $\sqrt{\left\langle\theta^{2}\right\rangle} \sim \pi$. There are alternative-more physically realistic - bath distributions for which the Markovian limit arises naturally.

Drude dynamics. The Drude bath has density of states $J(\omega)=4 \gamma \omega \frac{\omega_{d}^{2}}{\omega^{2}+\omega_{d}^{2}}$, where the Drude frequency, $\omega_{d}$, defines the bath cutoff. The new energy scale allows the bath to be in its classical limit, $T \gg \omega_{d}$, even when the equilibrium thermal fluctuations of the system are small, $T \ll B$. It is then sensible to discuss the dissipation-dominated relaxation to this equilibrium distribution.

The Drude bath has a memory on timescales determined by the cutoff, $\omega_{d}^{-1}$. The dynamical dependence on the history of the bath is captured by introducing a time-dependent field term

$$
\begin{aligned}
\dot{\mathbf{s}}_{i} & =-\mathbf{s}_{i} \times\left(\mathbf{B}_{i}+\mathbf{B}_{\mathrm{diss}}\right), \\
\dot{\mathbf{B}}_{\mathrm{diss}} & =-\omega_{d}\left(\mathbf{B}_{\mathrm{diss}}-\gamma \hat{\mathbf{z}}\left[\hat{\mathbf{z}} \cdot\left(\mathbf{s}_{i} \times \mathbf{B}_{i}\right)\right]-\boldsymbol{\eta}\right),
\end{aligned}
$$

where $\left.\left\langle\eta_{z}(t) \eta_{z}\left(t^{\prime}\right)\right)\right\rangle=2 \gamma T \delta\left(t-t^{\prime}\right)$ and as before and we take $\omega_{d} \ll T$. This is shown in detail in Appendix B. The main effect of the bath is to induce oscillations in $\phi$ about the Markovian trajectory. In this strongly dissipative limit the oscillations have a frequency $\sqrt{\omega_{d} / \tau_{\phi}}$ and describe fluctuations that are small in the $\theta$ direction and decay away on the bath memory timescale $1 / \omega_{d}$. When the oscillation decay is much faster than the decay of $\theta$, i.e., $\omega_{d} \gg B / s \gamma$, we recover the Markovian $O(2)$ dynamics described by Eqs. (16). A derivation of this is shown in Appendix B. The characteristic qualities of a typical trajectory are depicted in Fig. 2, while a simulation of the dynamics of Eq. (18) showing the separation of characteristic timescales is shown in Figs. 3 and 4.

To conclude this discussion we summarize the physical limits we have studied. We have assumed that the equilibrium thermal fluctuations are small $B \gg T$ and that the environmental coupling is strong. We have further assumed that temperature prevents quantum correlations persisting in the bath, $T \gg \omega_{d}$, though this condition may be relaxed. Our analysis shows an effective reduction in phase space from $O(3)$ to $O(2)$ resulting from the confinement of the $\phi$ coordinate to a typically small region around $\phi=0$. This occurs when $B \gg T \gg \omega_{d} \gg B / \gamma s$ for both Markovian and Drude dynamics. In this parameter regime we have also shown that when coupled to the more general Drude bath, the system is described by the Markovian $O(2)$ dynamics of Eq. (16) on timescales greater than the bath memory, $1 / \omega_{d}$.

\section{CONSEQUENCES OF ANISOTROPIC DISSIPATION}

The Langevin equation derived in the previous section exhibits markedly distinct behaviors in different regimes. This has implications both for attempts to fit experimental data to such models and, ultimately, for the usefulness of a system described by them for computation.

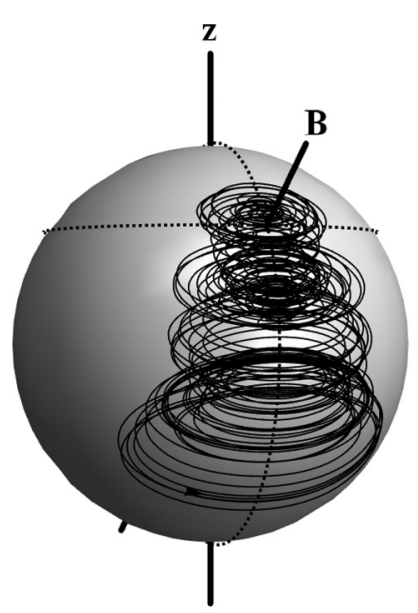

(a)

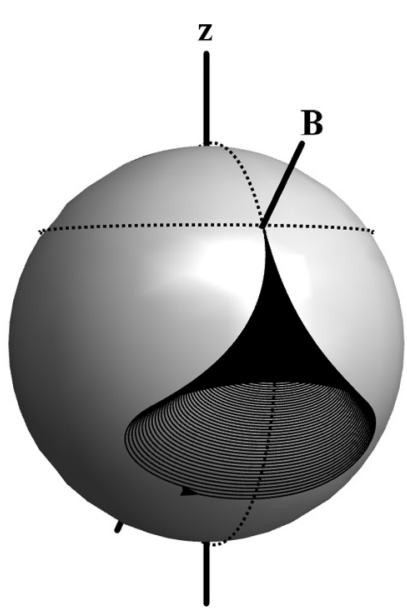

(b)
FIG. 2. Typical oscillatory stochastic dynamics of spins in the presence of anisotropic dissipation with a Drude bath. (a) A trajectory (solid) with parameters $\gamma=400, B=50 / 3 T=50 \omega_{d}$, plotted on the Bloch sphere. (b) The same trajectory plotted with the unphysical choice of $T=0$ to illustrate the deterministic part of the dynamics: The system oscillates around the $O(2)$ manifold with decaying amplitude. The lines $\phi=0$ and $\theta=\theta^{*}$ (dashed) are shown, and an arrowhead indicates the initial state.

\section{A. Different behaviors for the same system}

For a qubit to be useful for quantum computation it must be sufficiently manipulable. For the simplest system of a single qubit, this in effect requires the implementation of at least two nonparallel magnetic fields. The field, $\mathbf{B}$, is thus assumed to be a tunable parameter of the system, whereas the environmental coupling $\gamma$ is fixed at some finite value.

For the anisotropic coupling to the environment studied here, the strength of dissipation depends not only upon the fixed parameter $\gamma$, but also upon the orientation of the magnetic field, $\mathbf{B}$. This is important when characterizing such a device. In particular a system that is analyzed under conditions when $\mathbf{B}$ and $\mathbf{z}$ are nearly aligned will appear weakly coupled, whereas for other orientations of $\mathbf{B}$ the dynamics may be entirely dominated by environment-induced dissipative dynamics.

It is a general feature of qubit systems that inhomogeneous environmental couplings will result in dynamics that are correspondingly inhomogeneous $[3,12,29,33]$. We see here that certain states evolve with minimal dissipation, while others are dominated by dissipative or noisy dynamics. In extreme cases this may amount to a reduction in the effective state space.

\section{B. A model for lossy qubit arrays}

A natural application of our analysis is to understand some puzzling features of the D-wave machine [41]. This machine consists of a tuneable array of coupled Josephson junctions whose dynamics may be controlled to perform a quantum annealing or adiabatic computation. Various models have been posited for this system, including Bloch-Redfield simulations, Landau-Lifshitz-Gilbert models and two different 


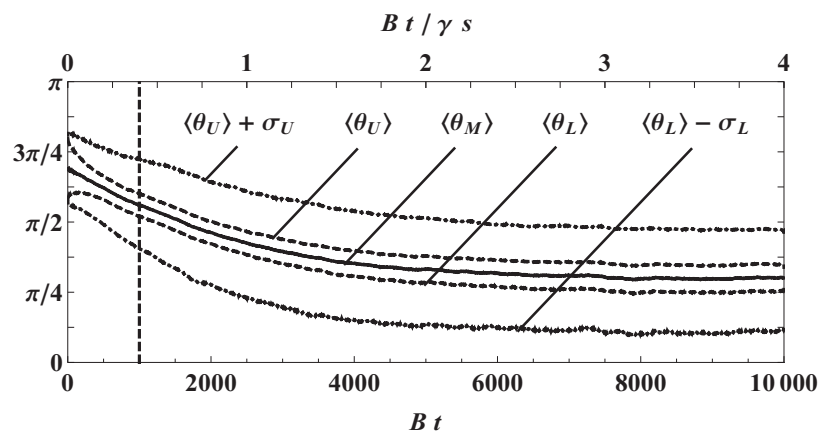

(a)

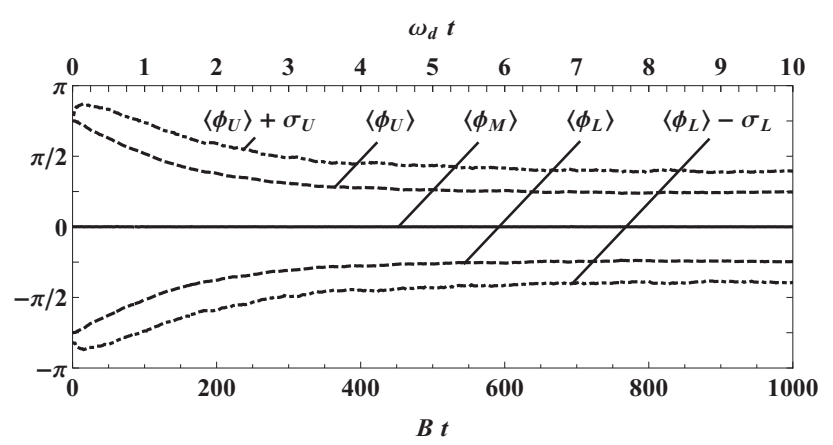

(b)

FIG. 3. Different timescales of relaxation in the stochastic dynamics of (a) $\theta$ and (b) $\phi$ when coupled to a Drude bath (note the different plot ranges). An ensemble of 1000 spins $(s=1 / 2)$ initially at $\theta=3 \pi / 4, \phi=3 \pi / 4$ when $t=0$ evolve with a magnetic field in the $\theta^{*}=\pi / 4, \phi^{*}=0$ direction. The coupling is $\gamma=5 \times 10^{3}$, with energy scales $B=10 T=100 \omega_{d}$, satisfying $B \gg T \gg \omega_{d} \gg$ $B / \gamma s$. On the timescales of $\theta$ dynamics the fast oscillations in the trajectories [see Fig. 4(c)] means that the trajectories are best characterised by an envelope with upper bound $\theta_{U}$, lower bound $\theta_{L}$ and mid-point $\theta_{M}$, these are simply read off the oscillating trajectory as shown in Fig. 4(c). Since the initial conditions is an extrema of the fast oscillations the initial point lies on $\theta_{U}$ and $\phi_{U}$. The ensemble averages $\left\langle\theta_{M}\right\rangle$ (solid) and $\left\langle\theta_{U}\right\rangle,\left\langle\theta_{L}\right\rangle$ (dashed) are shown with $\left\langle\theta_{U}\right\rangle+\sigma_{U}=\left\langle\theta_{U}\right\rangle+\sqrt{\left\langle\theta_{U}^{2}\right\rangle-\left\langle\theta_{U}\right\rangle^{2}}$, and $\left\langle\theta_{L}\right\rangle-\sigma_{L}=$ $\left\langle\theta_{L}\right\rangle-\sqrt{\left\langle\theta_{L}^{2}\right\rangle-\left\langle\theta_{L}\right\rangle^{2}}$ (both dot-dashed) illustrating the ensemble width. (a) The slow $\theta$ coordinate relaxes towards the equilibrium value $\theta^{*}=\pi / 4$ on a timescale $\gamma s / B$, approaching it at $t \approx 10^{4} / B$. The vertical line indicates the range of plot (b). (b) The same statistics are presented for the $\phi$ dynamics: The $\phi$ dynamics relaxes to its equilibrium distribution much faster on a characteristic timescale $\tau \sim 1 / \omega_{d}$ and is fully relaxed by $t \approx 500 / B$.

$O$ (2) models (a thorough review can be found in Ref. [4]). Our analysis sheds light upon the relationship between these different models and the fact that apparently quite different models yield surprisingly similar results. In particular, the salient features of the two $O(2)$ models proposed in the underdamped [5] and overdamped [7,24] regimes can be found in different limits of the Landau-Lifshitz-Gilbert model when proper attention is paid to the effects of the bath. These models are appropriate in a limit where decoherence renders

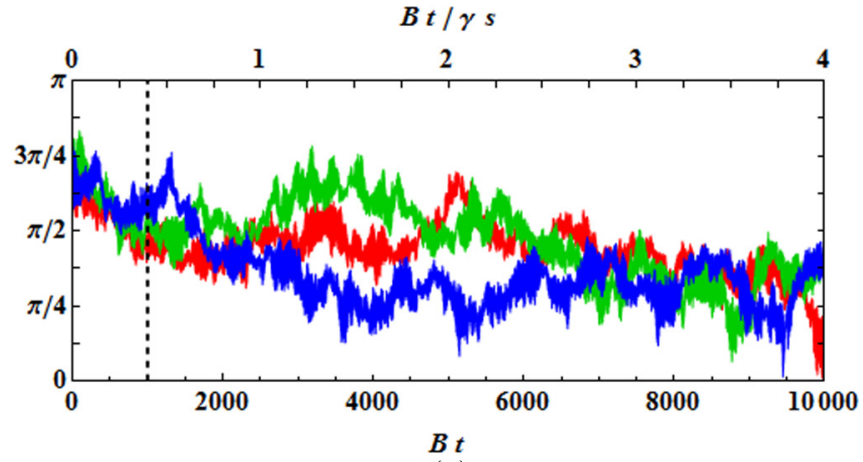

(a)

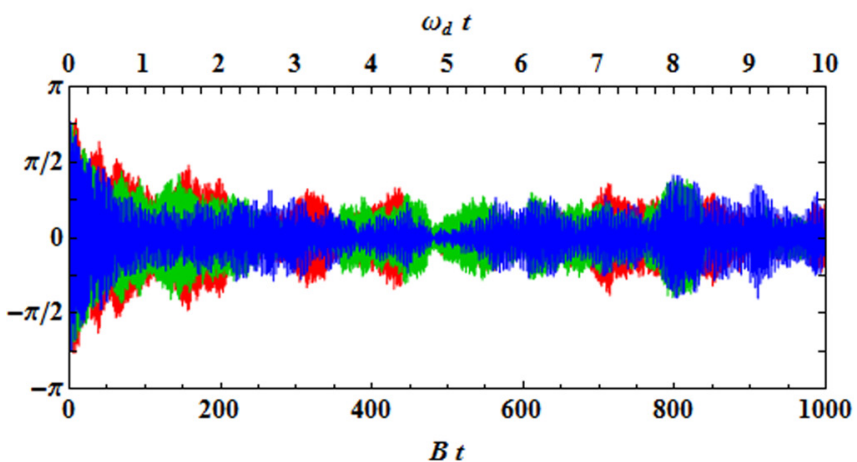

(b)

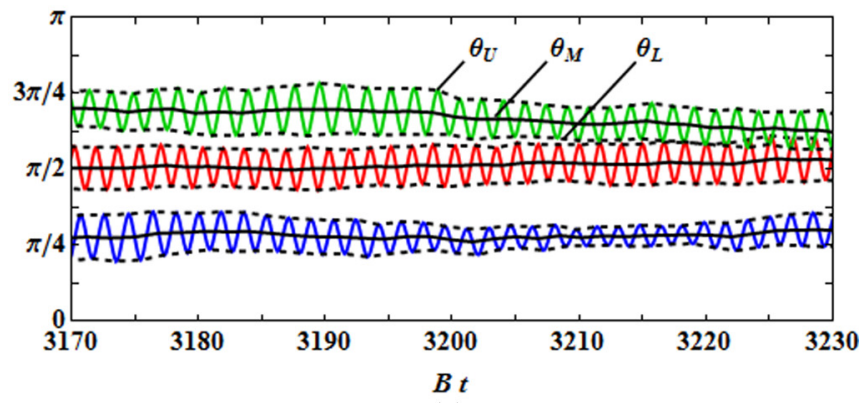

(c)

FIG. 4. Different dynamical timescales of typical trajectories in $\theta$ and $\phi$ when coupled to a Drude bath. A plot of three sample trajectories from the ensemble studied in Fig. 3. Being drawn from the ensemble these spins $(s=1 / 2)$ evolved in the same conditions: initially prepared at $\theta=3 \pi / 4, \phi=3 \pi / 4$ at $t=0$ and evolve with a magnetic field in the $\theta=\pi / 4, \phi=0$ direction. The coupling is $\gamma=5 \times 10^{3}$, with energy scales $B=10 T=100 \omega_{d}$, satisfying $B \gg$ $T \gg \omega_{d} \gg B / \gamma s$. (a) Trajectories in $\theta$ relax on a long timescale. The vertical dashed line indicates the range of plot (b). (b) $\phi$ relaxes on a shorter timescale, the confinement of $\phi$ is evidenced by the typically small excursions from $\phi=0$. (c) Oscillatory behavior induced by the bath occurring on shorter timescales $\tau \sim \sqrt{\tau_{\phi} / \omega_{d}}$ is plotted for $\theta$, similar behavior occurs for $\phi$. This behavior is expanded on in Appendix B, where the oscillations appear in Eq. (B5). Each oscillatory trajectory can be characterized by the upper and lower edges $\theta_{U}$ and $\theta_{L}$ of its envelope, and its midpoint $\theta_{M}$. The ensemble statistics of these quantities are studied in Fig. 3.

entanglement effects negligible. Bloch-Redfield simulationsthe only one of the above to include entanglement-are expected to yield similar results in this limit. 
In the underdamped limit, the qubit precesses about the adiabatic minimum. Projecting this motion onto the polar angle results in harmonic oscillations of the polar angle about its adiabatic minimum. This is essentially the $O(2)$ model of Ref. [5], though strictly the effective kinetic term depends upon the local effective field felt by the quantum bit and so varies through the computation [42]. This model is expected to accurately reflect the dynamics up to the point where it deviates markedly from adiabatic, accounting for its success in predicting the probability of correctly performing adiabatic computation in some circumstances [43].

In this paper we have focused upon the overdamped limit. A model of overdamped classical rotors undergoing a thermal exploration of the $O(2)$ state space was introduced in Ref. [7]. This model reproduced additional, apparently quantum, effects [23], though it is interesting that the underdamped nonthermal $O$ (2) model was also able to reproduce these results. Further statistics presented in evidence of quantum effects [4] were reproduced only by the overdamped classical model [24] and not the underdamped model. The overdamped model of Refs. [7] and [24] can be obtained by artificially confining the motion of overdamped classical $O(3)$ spins to the $O(2)$ phase space [44]. Our analysis in Sec. IV B shows that, remarkably, anisotropic dissipation can bias the dynamics towards just such a confinement: the $O(3)$ dynamics of dissipative spin dynamics [Eqs. (11) and (18)] are reduced to the effective $O(2)$ dynamics of Eq. (16) as illustrated in Figs. 3 and 4. An unanticipated feature of this relaxation to an $O(2)$ manifold is the restriction of the system dynamics to half of the $O(2)$ submanifold. The whole of this submanifold is a fixed point of the initial rapid decay, but half forms a stable and half an unstable fixed point.

The representation of overdamped classical rotors in Refs. [7] and [24] is at first glance rather different from the Langevin equation of Eq. (16). However, Metropolis-Hastings dynamics describes a dissipative relaxation to thermal equilibrium and has been used previously to model dynamics described by a Langevin equation [38-40,45]. The ad hoc model of Refs. [7] and [24] do not include the biasing of trajectories to half of the $O(2)$ manifold that naturally arises from microscopic considerations. However, the dynamics of the component of qubit projected onto the direction of the local field is rather similar (see Appendix C), perhaps accounting for the success of Refs. [7] and [24] despite their models not being strictly derivable from microscopic considerations.

These analyses raise an immediate question of whether the D-wave system is in an overdamped or underdamped limit. This is subtle. As discussed in Sec. VA, the strength of damping depends upon the microscopic details of coupling to the bath, the orientation of the effective field relative to the $z$ axis, and the instantaneous position of the qubit on the Bloch sphere. Because of the latter effects, the dissipation is largest at the start of D-wave computation, when the effective field and Bloch spins are in the $x y$ plane, and decreases to zero as the computation proceeds. Even when the coupling to the bath is strong, and the initial dynamics overdamped, there is a transition to underdamped dynamics at some point in the computation. Whether or not the overdamped or underdamped dynamics determines the success or failure of a computation depends upon precisely when this crossover occurs.
So is the D-wave system initially overdamped or underdamped? This question does not appear to be resolved by published experimental data. To our knowledge no direct measurements of $T_{1}$ or $T_{2}$ times on the D-Wave are available. Measurements of high-frequency flux noise via macroscopic resonant tunneling [46] indicate that above a cutoff $\omega_{\mathrm{HF}}=$ $0.5 \mathrm{GHz}$ noise is Ohmic with $\gamma_{\mathrm{HF}}=\Phi_{0}^{2} S_{\Phi}(0) / 8 \hbar k_{\mathrm{B}} T L^{2} \approx$ 0.5 , where the values for inductance $L=265.4 \mathrm{pH}$ and shunt resistance $R=2 k T L^{2} / S_{\Phi}(0)=20 k \Omega$ measured in Ref. [46], and $\Phi_{0}$ is the magnetic flux quantum. However, our analysis shows that the qubit is also sensitive to noise with frequency lower than the system frequency. At the lowest frequencies, the noise is of $1 / f$ form [47]. There is a large window between the high-frequency [46] and the low-frequency measurements [47] in which the noise has not been directly measured. The measured current noise characteristics do not, therefore, preclude the possibility of $\gamma>1$ and initial overdamped dynamics.

\section{CONCLUSION}

Our main result has been to show how anisotropic dissipation can bias quantum trajectories towards particular submanifolds of the system's Hilbert space. We have found a Langevin description of the dynamics of qubits that allows for anisotropic coupling to the environment. This is a natural generalization of the Landau-Lifshitz-Gilbert equations, which describe the dissipative dynamics of spins with isotropic coupling to the environment. The fluctuationdissipation relation has the important consequence that the anisotropic noise generated by this coupling inevitably leads to anisotropic dissipation.

This model applies explicitly to qubits experiencing dissipation due to fluctuations in the level separation (environmental coupling to the $\hat{s}_{z}$ operator). When the coupling to the bath is strong the anisotropic dissipation drives rapid relaxation to a reduced $O(2)$ manifold of constrained dynamics.

This emergence of this effective dynamics from the underlying microscopics reproduces some salient features of the dynamics of the models of Refs. [7,24]. These models were capable of reproducing several observed behaviours of the D-Wave machine previously believed to evidence quantum dynamics. This highlights the necessity of understanding the dynamics in dissipative and strong coupling cases when interpreting the dynamics of an experimental system.

Entanglement, which we neglect here, is crucial for full quantum dynamics, and necessary to get the exponential scaling between the quantum state space and number of qubits. It has been argued recently in Refs. [25,26] to act as a resource for adiabatic computation. These works modeled quantum adiabatic computation with artificial constraints on the entanglement analogous to the artificial constraint of a local subsystem to an $O(2)$ manifold. However, one may anticipate that the effects of dissipation could naturally bias the trajectories to these restricted manifolds. Understanding this will be key to determining how best to use limited or dissipating entanglement resources in computation.

Understanding of the effects of state-dependent noise and anisotropic coupling to the environment is crucial for the proper control of quantum devices. As we have shown in the case of the D-Wave machine, these effects can bias the system 
dynamics in unexpected ways. Used constructively, this may be harnessed to useful ends. If ignored, the dynamics may completely different from that intended.

\section{ACKNOWLEDGMENT}

This research was supported by the EPSRC under Grants No. EP/K02163X/1 and No. EP/I004831/2.

\section{APPENDIX A: STRONGLY DISSIPATIVE DYNAMICS}

In this appendix we derive the effective $O(2)$ dynamics given in Eqs. (16) and (17), in which the Markovian model features a confinement of $\phi$. In the dissipative limit $\tau_{p} \gg \tau_{d}$ the spin dynamics are given by Eq. (14). To leading order in the long timescale, the $\phi$ dynamics take the form

$$
\dot{\phi}=-s \gamma B \sin \theta^{*} \sin \theta \sin \phi+\eta=-\tau_{\phi}^{-1} \sin \phi+\eta,
$$

where as before $\tau_{\phi}^{-1}=\gamma s B \sin \theta \sin \theta^{*}$. This corresponds to a Fokker-Planck equation

$$
\frac{\partial p}{\partial t}=-\frac{\partial}{\partial \phi}\left(\frac{\sin \phi}{\tau_{\phi}}-\gamma T \frac{\partial}{\partial \phi}\right) p
$$

for an ensemble distribution $p$. The equilibrium solution to Eq. (A2) is given by

$$
p=\frac{\exp (A \cos \phi)}{2 \pi I_{0}(A)},
$$

where $A=\left(\gamma T \tau_{\phi}\right)^{-1}$ and $I_{n}(\cdot)$ are the modified Bessel functions of the first kind. Including the additional subleading terms [those in Eq. (14) that are missing from Eq. (A1)] leads to a Fokker-Planck equation with no closed-form solution. However, the salient features are captured by making an appropriate shift to $\phi$ and rescaling of $A$. This leads to an equilibrium solution

$$
\begin{aligned}
p & =\frac{\exp \left[A \cos \left(\phi-\phi^{*}\right)\right]}{2 \pi I_{0}(A)}, \\
\tan \phi^{*} & =\frac{\sin \left(\theta-\theta^{*}\right)}{s \gamma \sin ^{2} \theta \sin \theta^{*}},
\end{aligned}
$$

where $A=\left(\gamma T \tau_{\phi} \cos \phi^{*}\right)^{-1}$, which deviates from the exact solution only far from the distribution peak. In terms of $\theta, A$ takes the form $A=$ $B \sqrt{s^{2} \gamma^{2} \sin ^{4} \theta \sin ^{2} \theta^{*}+\sin ^{2}\left(\theta-\theta^{*}\right)} /(\gamma T \sin \theta)$.

Assuming a separation of timescales, the remaining $\theta$ dynamics can be found by averaging Eq. (14) over the equilibrium distribution of $\phi$ given in Eq. (A4). This leaves a single equation giving the $\theta$ dynamics,

$$
\dot{\theta}=-B \sin \theta^{*}\langle\sin \phi\rangle+\eta^{\prime},
$$

where $\theta$ is subject to a drift term originating from the mean value of the fast $\phi$ dynamics and a stochastic term, which originates from the fluctuations in the $\phi$ dynamics away from their mean value. This approximation becomes accurate on timescales $t \gg \tau_{\phi}$. Evaluating this exactly we obtain

$$
\langle\sin \phi\rangle=\int d \phi p(\phi) \sin \phi=\sin \phi^{*} \frac{I_{1}(A)}{I_{0}(A)}
$$

for the mean, whereas the noise term is defined by its mean $\left\langle\eta^{\prime}(t)\right\rangle=0$ and covariance

$$
\begin{aligned}
& \left\langle\eta^{\prime}(t) \eta^{\prime}\left(t^{\prime}\right)\right\rangle \\
& =\left(B \sin \theta^{*}\right)^{2}\left(\left\langle\sin \phi(t) \sin \phi\left(t^{\prime}\right)\right\rangle-\langle\sin \phi(t)\rangle\left\langle\sin \phi\left(t^{\prime}\right)\right\rangle\right) \\
& \approx\left(B \sin \theta^{*}\right)^{2} \exp \left(-\left|t-t^{\prime}\right| / \tau_{\phi}\right)\left(\left\langle\sin ^{2} \phi\right\rangle-\langle\sin \phi\rangle^{2}\right) \\
& \approx 2 \tau_{\phi}\left(B \sin \theta^{*}\right)^{2} \delta\left(t-t^{\prime}\right)\left(\left\langle\sin ^{2} \phi\right\rangle-\langle\sin \phi\rangle^{2}\right) \\
& =2 \tau_{\phi}\left(B \sin \theta^{*}\right)^{2} \delta\left(t-t^{\prime}\right)\left[\sin ^{2} \phi^{*}\left(1-\frac{I_{1}(A)}{A I_{0}(A)}-\frac{I_{1}^{2}(A)}{I_{0}^{2}(A)}\right)\right. \\
& \left.\quad+\cos ^{2} \phi^{*} \frac{I_{1}(A)}{A I_{0}(A)}\right] .
\end{aligned}
$$

We consider the limiting cases in which the above form simplifies: For $\boldsymbol{T} \ll \boldsymbol{B}$, we have $A \gg 1$ and $I_{1}(A) / I_{0}(A)=$ $1-1 /(2 A),+O\left(A^{-2}\right)$, which gives, to leading order

$$
\begin{aligned}
\langle\sin \phi\rangle & =\frac{\sin \left(\theta-\theta_{0}\right)}{s \gamma \sin ^{2} \theta \sin \theta^{*}}, \\
\left\langle\eta^{\prime}(t) \eta^{\prime}\left(t^{\prime}\right)\right\rangle & =2 \gamma T\left(B \tau_{\phi} \sin \theta^{*}\right)^{2} \delta\left(t-t^{\prime}\right) .
\end{aligned}
$$

For $\boldsymbol{T} \gg \boldsymbol{B}$, we have $A \ll 1$ and $I_{1}(A) / I_{0}(A)=A / 2+$ $O\left(A^{2}\right)$, which gives, to leading order in $\tau_{d} / \tau_{\mathrm{p}}$,

$$
\begin{aligned}
\langle\sin \phi\rangle & =\frac{B \sin \left(\theta-\theta_{0}\right)}{2 T \gamma \sin \theta}, \\
\left\langle\eta^{\prime}(t) \eta^{\prime}\left(t^{\prime}\right)\right\rangle & =\tau_{\phi}\left(B \sin \theta^{*}\right)^{2} \delta\left(t-t^{\prime}\right) .
\end{aligned}
$$

Substituting the values of Eqs. (A8) and (A9) into Eq. (A5) gives the forms Eqs. (16) and (17) respectively in the main body of the paper.

\section{APPENDIX B: DRUDE DYNAMICS}

In this appendix we derive the dynamical equation of a single spin coupled to a Drude bath and show that in an appropriate limit the long timescale $\theta$ dynamics are given by the Markovian equation (16) with $\phi$ remaining typically close to $\phi \approx 0$. Using the Drude density of states and evaluating Eqs. (9) and (10), in the limit of $T \gg \omega_{d}$, one obtains $\left\langle\eta(t) \eta\left(t^{\prime}\right)\right\rangle=T \gamma\left(t-t^{\prime}\right)=T \gamma \omega_{d} \exp \left(-\omega_{d}\left|t-t^{\prime}\right|\right)$. This particular noise $\eta(t)$ can be written in terms of a $\delta$-correlated stochastic dummy variable $\eta^{\prime}$ as

$$
\eta(t)=-\omega_{d} \int_{-\infty}^{t} d t^{\prime} \mathrm{e}^{-\omega_{d}\left(t-t^{\prime}\right)} \eta^{\prime}\left(t^{\prime}\right),
$$

which satisfies $\left\langle\eta^{\prime}(t) \eta^{\prime}\left(t^{\prime}\right)\right\rangle=2 \gamma T \delta\left(t-t^{\prime}\right)$. Rewriting Eq. (8) the dynamics are obtained as

$$
\begin{aligned}
\dot{\mathbf{s}}_{i} & =-\mathbf{s}_{i} \times\left[\mathbf{B}_{i}+\mathbf{B}_{\mathrm{diss}}(t)\right] \\
\mathbf{B}_{\mathrm{diss}}(t) & =-\omega_{d} \int_{-\infty}^{t} d t^{\prime} \mathrm{e}^{-\omega_{d}\left(t-t^{\prime}\right)}\left[\gamma \dot{s}_{i, z}\left(t^{\prime}\right)-\eta^{\prime}\right] \hat{\mathbf{z}} .
\end{aligned}
$$

Writing the second equation in its differential form we find

$$
\begin{aligned}
\dot{\mathbf{B}}_{\mathrm{diss}} & =-\omega_{d}\left(\mathbf{B}_{\mathrm{diss}}+\gamma \dot{s}_{i, z} \hat{\mathbf{z}}-\eta^{\prime} \hat{\mathbf{z}}\right) \\
& =-\omega_{d}\left\{\mathbf{B}_{\mathrm{diss}}-\gamma \hat{\mathbf{z}}\left[\hat{\mathbf{z}} \cdot\left(\mathbf{s}_{i} \times \mathbf{B}_{i}\right)\right]-\eta^{\prime} \hat{\mathbf{z}}\right\},
\end{aligned}
$$

where the second part follows by substituting $\dot{s}_{i, z}$ and noting that $\hat{\mathbf{z}} \cdot\left(\mathbf{s}_{i} \times \mathbf{B}_{\text {diss }}\right)=0$. These dynamics equate to Eq. (18) after trivial relabeling. 
Separating Eq. (B3) into the fast $\left(\phi, B_{\text {diss }}^{(z)}\right)$ and slow $(\theta)$ degrees of freedom and solving the linearised equations of motion for $\phi$ and $B_{\text {diss }}^{(z)}$ we find that

$$
\begin{aligned}
\dot{\theta} & =-B \sin \theta^{*}\langle\sin \phi\rangle+\eta^{\prime}, \\
\langle\sin \phi\rangle & =\frac{\sin \left(\theta-\theta_{0}\right)}{s \gamma \sin ^{2} \theta \sin \theta^{*}} \\
\left\langle\eta^{\prime}(t) \eta^{\prime}\left(t^{\prime}\right)\right\rangle & =\left(B \sin \theta^{*}\right)^{2} \operatorname{Cov}\left(t, t^{\prime}\right)
\end{aligned}
$$

as before in Eq. (A8). However, now there are longlasting oscillations in the noise, characterized by $\operatorname{Cov}\left(t, t^{\prime}\right)=$ $\left\langle\sin \phi(t) \sin \phi\left(t^{\prime}\right)\right\rangle-\langle\sin \phi(t)\rangle\left\langle\sin \phi\left(t^{\prime}\right)\right\rangle$. Evaluating this one finds that

$$
\begin{aligned}
\left\langle\eta^{\prime}(t) \eta^{\prime}\left(t^{\prime}\right)\right\rangle= & \gamma T \tau_{\phi}\left(B \sin \theta^{*}\right)^{2} \mathrm{e}^{-\frac{\omega_{d}\left|t t^{\prime}\right|}{2}} \\
& \times\left[\cos \left(\omega\left|t-t^{\prime}\right|\right)+\frac{\omega_{d}}{2 \omega} \sin \left(\omega\left|t-t^{\prime}\right|\right)\right],
\end{aligned}
$$

where $\omega=\sqrt{\frac{\omega_{d}}{\tau_{\phi}}\left(1-\frac{\omega_{d} \tau_{\phi}}{4}\right)}$ characterizes the oscillation frequency of the correlations. These fast oscillations can be seen in Fig. 4(c).

As expected on timescales much longer that $1 / \omega_{d}$ the covariance has the same value as with the previous cases

$$
\begin{aligned}
\left\langle\eta^{\prime}(t) \eta^{\prime}\left(t^{\prime}\right)\right\rangle & \approx 2 \gamma T\left(B \tau_{\phi} \sin \theta^{*}\right)^{2} \delta\left(t-t^{\prime}\right), \\
& =\frac{2 T}{\gamma(s \sin \theta)^{2}} \delta\left(t-t^{\prime}\right),
\end{aligned}
$$

thus on timescales much greater than the inverse bath frequency we recover the Markovian case [Eq. (A8)] and the $\theta$ dynamics of Eq. (16).

\section{APPENDIX C: ENSEMBLE DYNAMICS}

In this appendix we study the dynamics of an ensemble of noninteracting spins to show the appearance of the Markovian anisotropic dynamics at ensemble level. The ensemble dynamics are captured by a Fokker-Planck equation, which is found to have similarly anisotropic dynamics, which can in turn be related to Metropolis-Hastings dynamics.

We show that in the simplest case of noninteracting spins the ensemble dynamics do not agree with the Bloch equations; this is clear as the anisotropic nature of the dynamics persists even after the ensemble averaging. This result is perhaps surprising so we provide an explicit derivation from the Fokker-Planck equation.

Within the first moment approximation the ensemble is described by the probability distribution

$$
p(\mathbf{s}, t)=\frac{1}{Z} \mathrm{e}^{\xi(t) \cdot \mathbf{s}}, \quad Z=\frac{4 \pi}{\xi} \sinh (\xi s) .
$$

The first moment approximation in not appropriate when considering systems of interacting spins as correlations between the trajectories of different spins cannot be neglected. Thus, for the purpose of deriving ensemble dynamics, we consider a system of many spins without interactions between them, each acting under the influence of an external field $\mathbf{B}$ and coupled anisotropically, along the $\hat{\mathbf{z}}$ direction only, to an ohmic bath.

For a specific realization of the history of the bath, the dynamics are given by the Eq. (11b). When we sum over

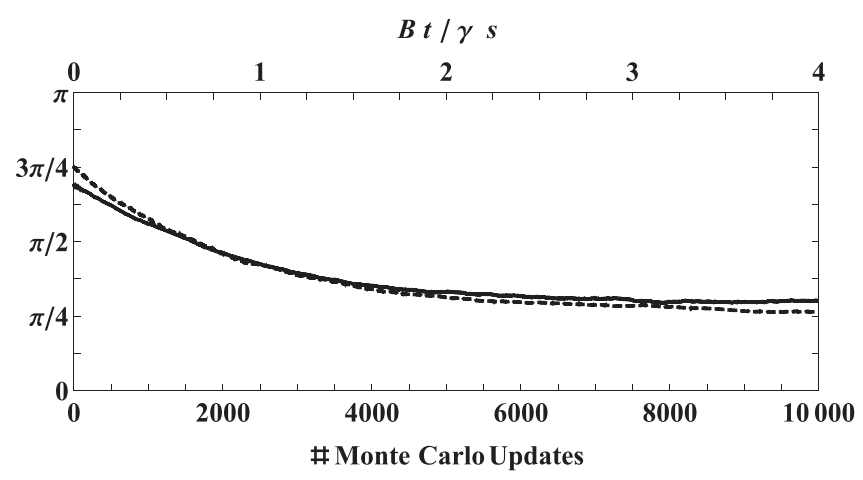

FIG. 5. Comparison of Metropolis-Hastings and anisotropic LLG dynamics. The ensemble averaged value $\langle\theta\rangle$ of Metropolis Hastings (dashed) dynamics is compared with the anisotropic LLG dynamics (solid) data from Fig. 3. The LLG dynamics have small oscillations (see Fig. 4 for further detail), thus we plot the ensemble averaged midpoint of these small oscillations, $\left\langle\theta_{M}\right\rangle$, as previously in Fig. 3. Both simulation were performed using an ensemble of 1000 spins ( $s=1 / 2$ ) initialised at $\theta=3 \pi / 4$, evolving in a magnetic field in the $\theta^{*}=\pi / 4, \phi^{*}=0$ direction, and a temperature $T=B / 10$. For the LLG dynamics the coupling is $\gamma=5 \times 10^{3}$, with energy scales $B=10 T=100 \omega_{d}$, satisfying $B \gg T \gg \omega_{d} \gg B / \gamma s$.

the histories of the bath, the dynamics are described by the evolution of the probability distribution $(\mathrm{C} 1)$.

Following the approach of Refs. [16,19]—in which an analogous calculation is performed for an isotropic bath coupling, i.e., microscopic dynamics corresponding to Eq. (1b)—we find that the evolution of $p(\mathbf{s}, t)$ is described by the Fokker-Planck equation

$$
\dot{p}=\nabla_{\mathbf{s}} \cdot\left(\mathbf{s} \times \mathbf{B}+\gamma \mathbf{S} \times \hat{\mathbf{z}}\left\{\hat{\mathbf{z}} \cdot\left[\mathbf{S} \times\left(\mathbf{B}-T \nabla_{\mathbf{s}}\right)\right]\right\}\right) p .
$$

By substituting Eq. (C1) into Eq. (C2) and integrating over s, the dynamics of the parameter $\xi=\xi \hat{\xi}$ are found to be governed by the equation

$$
\begin{aligned}
\dot{\xi} & +\xi \times \mathbf{B}+\gamma s^{2}\left(\frac{s}{\sigma}-\frac{3}{\xi s}\right) \xi \times \hat{\mathbf{z}}[\hat{\mathbf{z}} \cdot(\xi \times \mathbf{B})] \\
& +\gamma T\left[\left(\frac{\sigma}{\xi \sigma^{\prime}} \hat{\xi} \hat{\xi}^{T}\right)+\left(\mathbf{1}-\hat{\boldsymbol{\xi}} \hat{\xi}^{T}\right)\right]\left(\mathbf{1}-\hat{\mathbf{z}} \hat{\mathbf{z}}^{T}\right)\left(\boldsymbol{\xi}-\boldsymbol{\xi}_{0}\right)=0 .
\end{aligned}
$$

The fixed point of the dynamics is given by $\boldsymbol{\xi}_{0}=\mathbf{B} / T$, which corresponds to the Boltzmann distribution. $\boldsymbol{\sigma}=\langle\mathbf{s}\rangle$ is the mean polarization vector with norm $\sigma=\frac{1}{Z} \frac{d Z}{d \xi}=s\left(\operatorname{coth}(\xi s)-\frac{1}{\xi s}\right)$ and $\sigma^{\prime}=\frac{d \sigma}{d \xi}$ its derivative.

The physical origins of the first three terms are clear, as they correspond to the respective terms of Eq. (11b). The last term corresponds to longitudinal relaxation with a rate $\Gamma_{1}=\gamma T$ and transverse relaxation with a rate $\Gamma_{2}=\frac{\gamma T \sigma}{\xi \sigma^{\prime}}$. It should be noted, however, that the dynamics described by these terms differs from the usual isotropic case due to the projecting out of the component in the $\hat{\mathbf{z}}$ direction, this reflects the underlying anisotropy of the coupling.

It is possible to rewrite Eq. (C3) to give the dynamics of the ensemble polarization $\sigma$, where $\xi$ is defined implicitly by the 
form of $\sigma(\xi)$. Doing so, one obtains

$$
\begin{aligned}
\dot{\boldsymbol{\sigma}} & +\boldsymbol{\sigma} \times \mathbf{B}+\gamma \frac{s^{2}}{\sigma^{2}}\left(1-\frac{3 \sigma}{\xi s^{2}}\right) \boldsymbol{\sigma} \times \hat{\mathbf{z}}[\hat{\mathbf{z}} \cdot(\boldsymbol{\sigma} \times \mathbf{B})] \\
& +\gamma T\left(\mathbf{1}-\hat{\mathbf{z}} \hat{\mathbf{z}}^{T}\right)\left(\sigma-\frac{\sigma}{\xi T} \mathbf{B}\right)=0 .
\end{aligned}
$$

For a highly polarized ensemble, $\sigma \approx s$, at low temperatures, the ensemble dynamics converge upon the microscopic dynamics of Eq. (11b) showing that the unique behaviors described in the body of the paper persist in the ensemble dynamics of the system.
These ensemble dynamics are shown in Fig. 3 where they are obtained from sampling the stochastic sampling of the trajectories defined by Eq. (11), a sample of these trajectories is shown in Fig. 4. In the main text we discuss the similarity between these dynamics and the MetropolisHastings dynamics of Refs. [7] and [24]. This similarity is most evident in their confinement to dynamics on an $O(2)$ manifold, but is clear also in the similarity between their dynamics, both of which are overdamped and dissipative, as shown in Fig. 5. The existence of such a relationship between overdamped spin dynamics and the Metropolis-Hastings algorithm has been previously established [38-40].
[1] L. D. Landau and E. Lifshitz, Phys. Z. Sowjetunion 8, 101 (1935).

[2] T. L. Gilbert, IEEE Trans. Magn. 40, 3443 (2004).

[3] A. J. Leggett, S. Chakravarty, A. T. Dorsey, M. P. A. Fisher, A. Garg, and W. Zwerger, Rev. Mod. Phys. 59, 1 (1987).

[4] T. Albash, W. Vinci, A. Mishra, P. A. Warburton, and D. A. Lidar, Phys. Rev. A 91, 042314 (2015).

[5] J. A. Smolin and G. Smith, Frontiers Phys. 2 (2014).

[6] L. Wang, T. F. Rønnow, S. Boixo, S. V. Isakov, Z. Wang, D. Wecker, D. A. Lidar, J. M. Martinis, and M. Troyer, arXiv:1305.5837.

[7] S. W. Shin, G. Smith, J. A. Smolin, and U. Vazirani, arXiv:1401.7087.

[8] A. Kamenev, Field Theory of Non-Equilibrium Systems (Cambridge University Press, Cambridge, 2011).

[9] P. P. Orth, A. Imambekov, and K. Le Hur, Phys. Rev. A 82, 032118 (2010).

[10] A. G. Green, Phys. Rev. B 73, 140506 (2006).

[11] A. Schmid, J. Low Temp. Phys. 49, 609 (1982).

[12] U. Weiss, Quantum dissipative systems, Vol. 10 (World Scientific, Singapore, 1999).

[13] R. Kubo and N. Hashitsume, Prog. Theor. Phys. Suppl. 46, 210 (1970).

[14] P. C. Hohenberg and B. I. Halperin, Rev. Mod. Phys. 49, 435 (1977).

[15] A. Jayannavar, Zeitschrift für Physik B Condensed Matter 82, 153 (1991).

[16] D. Garanin, V. Ishchenko, and L. Panina, Theor. Math. Phys. 82, 169 (1990).

[17] D. Garanin, Physica A 172, 470 (1991).

[18] T. Plefka, Zeitschrift für Physik B Condensed Matter 90, 447 (1993).

[19] D. A. Garanin, Phys. Rev. B 55, 3050 (1997).

[20] Z. Nussinov, A. Shnirman, D. P. Arovas, A. V. Balatsky, and J. X. Zhu, Phys. Rev. B 71, 214520 (2005).

[21] H. Katsura, A. V. Balatsky, Z. Nussinov, and N. Nagaosa, Phys. Rev. B 73, 212501 (2006).

[22] E. Chudnovsky, D. Garanin, and M. O'Keeffe, J. Superconductivity Novel Magnetism 25, 1007 (2012).

[23] S. Boixo, T. F. Rønnow, S. V. Isakov, Z. Wang, D. Wecker, D. A. Lidar, J. M. Martinis, and M. Troyer, Nat. Phys. 10, 218 (2014).
[24] S. W. Shin, G. Smith, J. A. Smolin, and U. Vazirani, arXiv:1404.6499.

[25] P. J. D. Crowley, T. Đurić, W. Vinci, P. A. Warburton, and A. G. Green, Phys. Rev. A 90, 042317 (2014).

[26] B. Bauer, L. Wang, I. Pižorn, and M. Troyer, arXiv:1501.06914.

[27] Neglecting zero-point fluctuations of the environment this reduces to the time domain form $\left\langle\eta_{\alpha}(t) \eta_{\beta}\left(t^{\prime}\right)\right\rangle=2 \gamma T \delta_{\alpha \beta} \delta\left(t-t^{\prime}\right)$.

[28] R. McDermott, IEEE Trans. Appl. Supercond. 19, 2 (2009).

[29] N. Prokof'ev and P. Stamp, Rep. Prog. Phys. 63, 669 (2000).

[30] L. Faoro and L. B. Ioffe, Phys. Rev. Lett. 96, 047001 (2006).

[31] R. de Sousa, Phys. Rev. B 76, 245306 (2007).

[32] A. Shnirman, Y. Makhlin, and G. Schön, Phys. Scr. T102, 147 (2002).

[33] M. Dubé and P. Stamp, Chem. Phys. 268, 257 (2001).

[34] F. Yoshihara, K. Harrabi, A. O. Niskanen, Y. Nakamura, and J. S. Tsai, Phys. Rev. Lett. 97, 167001 (2006).

[35] M. H. S. Amin, C. J. S. Truncik, and D. V. Averin, Phys. Rev. A 80, 022303 (2009).

[36] As shown in Appendix $C$ these differences also exist at the ensemble level.

[37] J. P. Garrahan, R. L. Jack, V. Lecomte, E. Pitard, K. van Duijvendijk, and F. van Wijland, J. Phys. A: Math. Theor. 42, 075007 (2009).

[38] K. Kikuchi, M. Yoshida, T. Maekawa, and H. Watanabe, Chem. Phys. Lett. 185, 335 (1991).

[39] U. Nowak, R. W. Chantrell, and E. C. Kennedy, Phys. Rev. Lett. 84, 163 (2000).

[40] X. Z. Cheng, M. B. A. Jalil, H. K. Lee, and Y. Okabe, Phys. Rev. Lett. 96, 067208 (2006).

[41] M. Johnson, M. Amin, S. Gildert, T. Lanting, F. Hamze, N. Dickson, R. Harris, A. Berkley, J. Johansson, P. Bunyk et al., Nature (London) 473, 194 (2011).

[42] It can be derived in a spin-wave-like expansion, integrating out the components that do not lie in the great circle traversed by the adiabatic minimum.

[43] S. Boixo, T. Albash, F. M. Spedalieri, N. Chancellor, and D. A. Lidar, Nat. Commun. 4 (2013).

[44] T. Albash, T. Rønnow, M. Troyer, and D. Lidar, Eur. Phys. J. Special Topics 224, 111 (2015).

[45] The relation of the Metropolis algorithm to Fokker-Planck diffusion has been noted previously in general terms in Ref. [38] while 
other Monte Carlo methods have been specifically connected with Langevin-type dynamics in Refs. [39,40].

[46] T. Lanting, M. H. S. Amin, M. W. Johnson, F. Altomare, A. J. Berkley, S. Gildert, R. Harris, J. Johansson, P. Bunyk,

E. Ladizinsky, E. Tolkacheva, and D. V. Averin, Phys. Rev. B 83, 180502 (2011).
[47] R. Harris, J. Johansson, A. J. Berkley, M. W. Johnson, T. Lanting, S. Han, P. Bunyk, E. Ladizinsky, T. Oh, I. Perminov, E. Tolkacheva, S. Uchaikin, E. M. Chapple, C. Enderud, C. Rich, M. Thom, J. Wang, B. Wilson, and G. Rose, Phys. Rev. B 81, 134510 (2010). 